\title{
A New Approach to an Automated Air Traffic Control
}

\author{
Patchev Dragoljub \\ M-NAV (Macedonian Air Navigation Service Provider), Skopje 1000, Macedonia
}

\begin{abstract}
This paper identifies areas of improvements of the air traffic control system and proposes modification of the concept of automation by using available technologies. With the proposed modification, the current Europe wide en route network structure can be modified in order to make routes more optimal. For this new route network structure, a new concept of automation will be used to manage with the air traffic. The first identified area of improvement is implementation of automation process that will enable decentralization of the air traffic control functionality to each individual aircraft and this will be achieved through automated routing of the aircrafts and CD\&R (conflict detection and resolution). The FMS (flight management system) at the aircraft will make decisions for the optimal flight route based on the sensor inputs, information on selection of the routes, next hope points and flight levels, all received by ADS-B (automatic dependant surveillance-broadcast). The second area is processing the information about the deviation from the optimal route as in flight plan due to a traffic management (vectoring, level change) and taking it into consideration when further actions are undertaken. For each action, a cost factor will be calculated from the fuel burned for that action. This factor will be used to select conflict resolution protocol. The proposed concept shall increase the capacity of the network, and enable the air traffic more efficient and more environmentally friendly while maintaining safe separation.
\end{abstract}

Key words: Automated air traffic control, ADS-B, efficient flight.

\section{Introduction}

The entire airspace used for civil flights is segmented into elementary volumes defining elementary sectors with limited capacity in terms of serving finite number of aircrafts per hour. There is one airway ${ }^{1}$ or more in each elementary sector that connects entry/exit points of that sector. In order to increase capacity, more sectors will be required. Creation of new sectors can not be extended unlimitedly. Safe en route traffic in core area of Europe is ensured by complex ground systems, procedures and skilled air traffic controllers. Each of the three mentioned elements is in a closed loop permanently monitoring and managing safe air traffic. The safe air traffic requires conflict free traffic, and the term conflict free means aircrafts can remain separated, e.g., at least $5 \mathrm{NM}$ laterally and $1,000 \mathrm{ft}$ vertically. Currently, flight management system

Corresponding author: Patchev Dragoljub, M.Sc., engineer, research field: application of information technology in aviation. E-mail: patcev@yahoo.com.

${ }^{1} \mathrm{~A}$ control area or portion thereof established in the form of a corridor (ICAO Annex 11-Air Traffic Services). installed in the modern aircraft calculates its lateral and vertical navigation and might be coupled with the autopilot and steering commands. It also holds flight plan which is entered by pilots prior to take-off. FMS (flight management system) uses different sensors to calculate aircraft position and determine optimum speed and level for given conditions. In one typical scenario, aircraft follows its flight plan but also makes deviation by manual modification of the route made by pilots on their decision or on request by air traffic controllers. Until now, only the ATC (air traffic control) center has had the traffic information mainly obtained through the existing radar systems. Having the full traffic situation awareness, the air traffic controllers are responsible for providing safe air traffic flow by permanently monitoring the traffic and giving clearances to the aircrafts for their routes. The current technologies provide sufficient information to the pilots and on board systems so they also can have sufficient information for the relevant traffic flow. The current centralized role of the air traffic controllers can be transferred and distributed to the 
pilots and on board systems. Several recent papers have focused on automation of the air traffic control, especially in solving CD\&R (conflict detection and resolution) and optimal routing problems but mainly in the terminal area. At present, both conflict resolution and optimal routing remain mostly disconnected as it is concluded in Ref. [1], the two areas of research as routing methods do not incorporate conflicts inherently in their problem formulation. In the paper, it is taken a step towards bringing optimal routing and conflict resolution closer together. In particular, the paper is inspired from routing methods in optical communication networks, where optical packets cannot be buffered and must therefore continuously move within the network without colliding. The optimization criterion based on a Floyd-Warshall shortest path algorithm is presented in Ref. [2]. It gives the shortest path between every pair of points on the grid. The authors propose the criteria but also some limitations should be added in order to provide a route network which could be potentially valid in reality: (1) The distance between two crossing points cannot be smaller than 100 kilometers so that an aircraft can spend more time on a main trunk than in a crossing area; (2) The shortest path cannot include an angle smaller than $90^{\circ}$ for making turns points feasible. As the criteria favor main flows, some flows (which are mainly orthogonal to these major flows) with only a few airplanes a day may have awkward trajectories with turning angles impossible to manage in reality. The computational agents that can control simulated air traffic as an attractive complement to traditional human-in-the-loop simulation methods, and safety/risk assessment are proposed in Ref. [3]. In the paper, the authors have elaborated a case study in which computational models of Terminal-Area air traffic controllers were evaluated against human performance on the same simulated traffic scenarios.

This paper is organized as follows. In Section 2, the problem addressed in this paper is briefly described.
Section 3 gives a detailed concept description. Section 4 explains calculation of the cost index and second area of improvement. Section 5 addresses operational aspects that will need to be considered in order to make the proposed concept operationally feasible. Section 6 provides brief summary and conclusions.

\section{Problem Definition}

The roots of today's concept of air traffic control go back to the middle of last century when surveillance radars were introduced for civil aviation. With the radars, air traffic controllers are able to see and track the aircrafts, and manage air traffic based on that information. Even with the application of the powerful computers and software today, basic concept is still the same. But the current technologies as technical enabler for safe air traffic control provide more sufficient information for both the pilots and the airborne systems in order to support them in making decisions on the most optimal route. Part of the current role of the air traffic controllers can be transferred to the pilots and on board systems. In this context, the purpose of this paper is to propose humanly monitored concept according to which, on board systems and pilots, would be decision makers and the air traffic controllers would only be supervisors of the actions, serving as backup and last resort part of the system. In this paper, a concept that will enable automated route selection and safe separation from the other traffic performed only by airborne systems is proposed. The criterion for the selection of optimal route is cost based. The key parameter for cost calculation is quantity of fuel burned on route.

\section{Concept Description}

In the initial concept definition, the focus is only on en route traffic in the upper area where aircrafts operate mostly in level flight and with constant speed. Finite part of the airspace with its entry and exit points is defined. The airspace is modeled with a graph 
model consisting of nodes that represent waypoints and links between nodes representing unidirectional airways with their distance, as shown in Fig. 1.

There is no restriction on the branching factor or number of the nodes. For example, if an airplane at node A should fly to node $\mathrm{J}$, the first task is to determine the "cheapest" available conflict free route. In the automation that is proposed, each aircraft will have a route network map as predefined in their automation system. As a first step, the airplane should find available path from node A to node J. This could be performed using a popular method by Dijkstra [4], the shortest path algorithm from the fifties, widely used in computer networks. Initially, the distance between nodes will be represented as a cost parameter of that link. Then the cost parameter could be slightly modified due to other factors like wind or speed. Head wind will increase cost, while tail wind will decrease it. When the available route is found (e.g., A-E-J), as a second step, the system will check for possible conflict situation during the timeline of the flight between the nodes. For that purpose ADS-B (automatic dependant surveillance-broadcast) messages can be used. Each ADS-B equipped aircraft periodically broadcasts its identity, position, and next trajectory changing point. The data obtained by ADS-B in system will be kept in each aircraft as its own data record for the position of the neighboring traffic. That record will be regularly updated. Update rate could be 1-3 s. This will provide sufficient time for position calculation.
This position update rate is improved when compared to the existing information obtained through secondary surveillance radar and tracker systems where typical refresh data rate is about $4 \mathrm{~s}$. With simple calculation of the trajectories and the known present position, next position and speed, the system can easily check whether any conflicting traffic exists on the particular route in the determined timeframe. If the path is conflict free, then this trajectory will be selected for the aircraft to fly from node $\mathrm{A}$ to node $\mathrm{J}$ and that information will be broadcasted so other aircrafts can update their own data record. If a possible conflict is detected during the calculations in the second process, then several options are available. Selecting the optimal option is determined by the "cost" of each action for the particular scenario. There are lots of papers describing different algorithms for conflict resolutions [5-7]. For selecting the conflict resolution procedure in each particular case, cost base criterion is proposed. Estimated cost is the amount of fuel burned for applying conflict resolution procedure. To perform the cost calculation, Eurocontrol BADA (base of aircraft data) [8] tables are used. BADA provides characteristic aircraft performance data including typical climb, descent speeds and vertical speeds (climb rates) as well as cruise speeds for operations within the ATC environment together with the amount of fuel burned for each phase of the flight. The data are organized in tables for many different aircraft types. For the purpose of possible

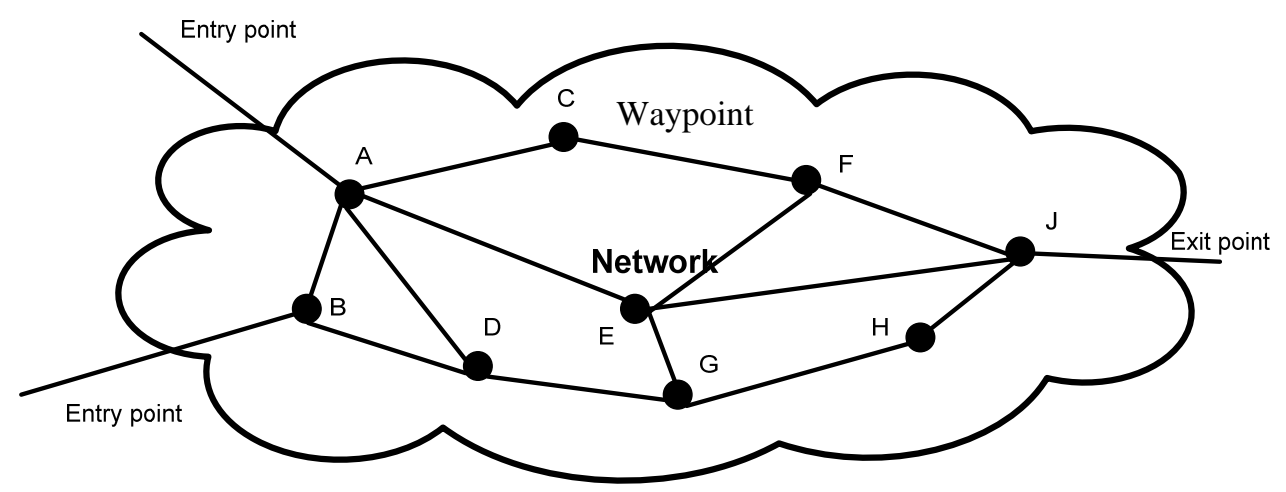

Fig. 1 Graph model of the airspace. 
conflict resolution, with simple calculation, the required quantity of fuel can be obtained for the aircraft to:

- fly extra distance to the new temporary added node and vector it back to the initial route (Fig. 2);

- change flight level;

- fly longer route, e.g., the second or third cheapest route found with Dijkstra shortest path algorithm during initial processing (A-C-E-F-J or A-D-G-H-J).

The comparison between amounts of fuel required for flying extended track (in case of vectoring) or level change and for flying longer route will determine the preferable conflict resolution option.

The proposed concept is modeled in MATLAB. For that purpose, a set of functions are created in MATLAB. The flow diagram is shown in Fig. 3. The functionalities of the simulator modeled in MATLAB in normal operation would be a part of the FMS in each aircraft.

For each leg between entry and exit point of the network functions from the flow chart shall be performed. A function "f_ospf" is called first. Entry parameters for the function "f_ospf" are entry node, exit node and flight level where aircraft intends to fly. Dijkstra algorithm checks the network topology and outputs a list of possible routes with their cost. For the lowest cost route, "f_confcheck" function is called where for the given time frame between entry and exit time at nodes for each leg which is part of the route, the function checks whether conflict traffic exists. A separate table is regularly updated with the data "received" (in our case simulated data) from ADS-B messages for the neighboring traffic. The function "f_confcheck" queries in the table for the time frame between entry and exit point at the leg if there is a traffic which might be in conflict. If there is no conflicting traffic, then a leg is reserved and that information is broadcasted. If the conflict exists, then conflict resolution algorithm is applied.

\section{Cost Index Calculation}

It can be considered that the total cost per flight at a given range consists of a fixed cost, cost depending on flight time, and fuel cost:

$$
C=C_{0}+C T \times t+C F \times W F
$$

where,

$C=$ total cost per flight;

$C_{0}=$ fixed cost (flight crew cost, ATC cost, cyclic maintenance cost...);

$C T=$ time related cost (flight crew cost if paid per hour, aircraft depreciation...);

$$
\begin{aligned}
& t=\text { trip time; } \\
& C F=\text { fuel cost; } \\
& W F=\text { trip fuel. }
\end{aligned}
$$

In the airline industry, CI (cost index) is defined as a ratio of fuel costs to all time related operating costs for one flight.

$$
C I=f\{C T / C F\}
$$

CI is the parameter which is used to choose the Mach/Altitude profile, and should, with available technology in correlation with the proposed concept, be used to solve the route. It represents optimization

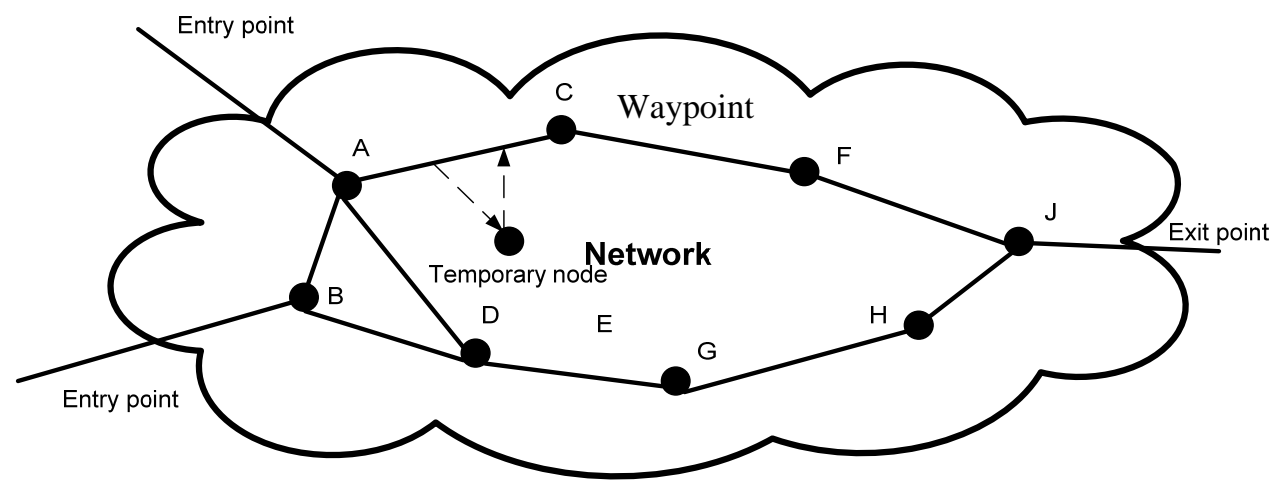

Fig. 2 Adding temporary node for conflict resolution. 


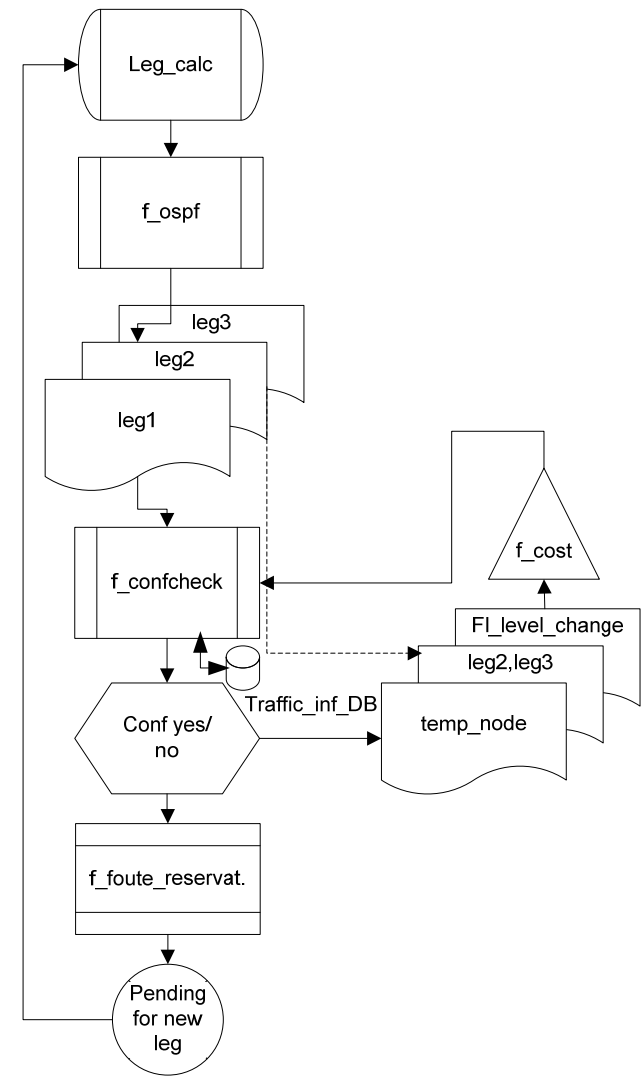

Fig. 3 Flow diagram.

parameter of the total trip cost by controlling both the fuel burned and the flight duration. The CI method calculation is a good parameter to be used for path optimization [9]. For short or medium-haul flights (like most in Europe), distances are short enough to allow aircrafts to carry and burn enough fuel to fly at maximal Mach and shorten the flight time. In this way, they make CT much lower than CF. For long-haul flights, a minimum burned fuel is an objective of the airliners so aircraft flies at such speed that will enable it to fly the longest distance. This makes CI minimal. For each flight, airliners calculate CI for each particular flight for given conditions and that determines flight profile balancing the time cost with a fuel cost for minimal total cost of operation. FMS installed in modern aircrafts can calculate optimal flight profile based on input value for CI. Companies select typical values of 80 fuel saving long haul flights or 250 to 500 for short haul flights when time related cost is significant.
With the current air traffic management, each individual air traffic unit/controller can modify aircraft trajectory and flight level. This will affect the optimal flight path, optimal flight level, speed or any combination of all of them. Any such action will change selected CI. Changing any of the flight parameters will prolong flight time (in case of new flight path longer than predicted) which will increase time related cost but also fuel related cost. Changing flight level, mostly will affect fuel related cost. Increasing the speed above the optimal will increase fuel related cost. The information for such changes from the optimal flight route is not distributed to the other ATC units. It can easily happen that one aircraft changes route or flight level due to requests from several air traffic control units many times during its flight. As a result of that, a yo-yo effect that will affect the aircraft will make the flying very inefficient. Within the automation, we propose a counter and weighted factor number to be used. The counter will increase its value for each change from the optimal flight route. Weighted factor number will be derived as a percentage of extra fuel burned for the longer path or flight level change, compared with fuel needed for the optimum flight profile. For each deviation from initial flight path, a new CI will be calculated. This information can be used when conflict resolution algorithm is performed. For the aircraft with lower counter values and lower weighted factor number, more "expensive" scenarios for resolution can be applied for the aircrafts with higher counter values and/or higher weighted factor number, less demanding conflict resolution scenarios or different conflict resolution scenario will be applied, e.g., other traffic will be re-routed. Counter value together with the weighted factor number will be distributed through the ADB-B messages to the neighboring aircraft, so each aircraft beside the position and next hope information for the neighboring traffic will hold information about "inefficiency” for each aircraft. 


\section{Operational Consideration}

For making fully operational concept, a modification to current ADS-B messages would be required in order to provide more information required for CD\&R algorithms as well as route reservation. For safety reasons, additional information distribution chain shall be established. Possible solution could be inclusion of low orbit satellites or ADS-B in ground stations which will also receive ADS-B messages from the aircrafts and forward the received data to the ground stations where a local data base system will be maintained and regularly updated with the traffic information. The information from the data base then shall be transmitted and aircrafts can compare their local data base with the one received from the ground stations.

\section{Conclusions}

The concept proposed in this paper extends the role of pilots and on board equipment. It enables delegating part of the air traffic controllers role to the pilots and the on board systems at the aircrafts. Initially the concept would be applicable only on en route traffic where airplanes are in horizontal flight most of the time. Several different algorithms are utilized to enable full automation. To find the most optimal route, the Dijkstra shortest path algorithm is used and then conflict check algorithm is applied. If there is no conflict, the selected route is reserved. And if the possible conflict exists, then conflict resolution algorithm is applied. The cheapest solution in terms of lowest quantity of fuel required for such a maneuver will be selected. The information about aircraft intention will be broadcasted and other participants will update their local data base for neighboring traffic. Position reports and next point information will be distributed by ADS-B. Some of the ADS-B messages would require modification or extension in order to make the concept feasible. A simulator that simulates described scenario is created in MATLAB in order to test the robustness of the concept and detect the possible weak points.

Furthermore, distribution of the information for the flight deviation from the optimal flight route due to an air traffic control management will enable selection of the most optimal conflict resolution algorithms for making flights more efficient and more environment friendly.

\section{References}

[1] Mishra, P., and Pappas, G. J. 2002. "Flying Hot Potatoes." In Proceedings of the American Control Conference, 754-9.

[2] Riviere, T., and Brisset, P. 2005. Shortest Path in Planar Graph and Air Route Network. EUROCONTROL Experimental Centre Innovative Research Activity report.

[3] Callantine, T. J. 2005. "Computational Modeling of Air Traffic Control: Terminal Area Case Study.” Presented at the 2005 IEEE International Conference: Systems, Man and Cybernetics, Hawaii, USA.

[4] Dijkstra, E. W. 1959. "A Note on Two Problems in Connection with Graphs.” Numerische Mathematik 1 (1): 269-71.

[5] Kuchar, J. K., and Yang, L. C. 2000. "A Review of Conflict Detection and Resolution Modeling Methods.” IEEE Transactions on Intelligent Transportation Systems 1 (4) : 179-89.

[6] Hwang, I., and Tomlin, C. J. 2007. "Protocol-Based Conflict Resolution for Air Traffic Control.” Air Traffic Control Quarterly 15 (1): 1-34.

[7] Spath, O., and Eyferth, K. 2001. "Conflict Resolution in En Route Traffic-A Draft Concept for an Assistance System Compatible with Solution of Air Traffic Controllers.”, MMI interaktiv 5 (7): 1-11.

[8] Eurocontrol. 2011. User Manual for the Base of Aircraft Data (BADA), Revision 3.9. EEC technical/scientific report.

[9] Sellier, F. L. 1990. "Discrete Real-Time Flight Plan Optimization.” S.M. thesis, MIT. 